\title{
Nonlinear Optics on a Silicon Platform for Broadband Light Generation and Ultrafast Information Processing
}

\author{
Bart Kuyken. Rayhael Van Laer, Francois Leo, Dries Vam \\ Thourhout, Roel Bacts, Gunther Roelkens \\ Photonics Research Group, Department of Information \\ Technology \\ Ghent University - imec \\ Ghent B-9000, Belgium \\ Bart.Kuyken@intec.ugent.be
}

Takuro Ideguchi, Simon Holzner, Ming Yan, Theodor W. Hänsch, Stéphane Coen, Peter Verheyen, Joris Van Campenhout, Simon-Pierre Gorza, Sylvain Combrie, Alfredo De Rossi, Fabrice Raineri, William M. J. Green, Nathalie Picqué,

\begin{abstract}
With the help of the strong nonlinear optical response in silicon nanophotonic wire waveguides complex sources and devices can be integrated on a chip. We demonstrate mid-infrared wavelength translators and frequency combs Furthermore, we open up the possibility for the integration of nonlinear optical functions at telecom wavelengths by exploiting more exotic nonlinear interactions in silicon waveguides as well as new waveguide piatforms.
\end{abstract}

Keywords Nonlinear opties, four-wave mixing; Nonlinear optics, integrated optics

\section{INTRODUCTION}

The high confinement of integrated waveguides, made out of high refractive index semi-conductor materials, can enhance nonlinear optical processes incredibly. Furthermore, the nonlinear refractive index of semi-conductor materials such as silicon or InGaP itself is very high. Hence, nonlinear optical interactions can become very efficient in semi-conductor waveguide platforms made out of these materials. These interactions can be used for a set of applications.

One application is the development of broadly tunable or broadband sources in the mid-infrared. These sources would meet the demand of sources needed for mid-infrared sensing. Mid-infared sensing exploits the strong and specific absorption lines of many molecules in the mid-infrared wavelength region [1], sometimes referred to as the "molecular fingerprint region". This would enable chip-scale optical sensors with a high sensitivity and selectivity. Through nonlinear optical mixing broadly tunable sources and devices can potentially be integrated on the silicon-on-insulator platform. Specifically, the absence of two-photon absorption beyond $2.2 \mu m$ - hampering efficient nonlinear interactions at telecom wavelengths - allows for efficient nonlinear interactions in the mid-infrared in silicon waveguides.
Another traditional application of nonlinear optics is ultrafast signal processing. However, because of the high two photon absorption at telecom wavelengths, crystalline silicon does not seem to be the ideal candidate here. Nonetheless, by shifting towards materials with a wider bandgap, two-photon absorption can be suppressed or eliminated at telecom wavelengths enabling efficient nonlinear interactions. We show that InGaP and a-Si:H on insulator nanowire waveguides can be used to obtain efficient nonlinear interactions in the telecom window with no or less parasitic two-photon absorption.

Lastly, we show that even in the presence of two-photon absorption at telecom wavelengths the photon-phonon interaction can be made efficient in silicon waveguides. The latter paves the way for making e.g. high quality all optical RF-filters on a CMOS platform.

All these demonstrations show how integrated platforms are very efficient for doing nonlinear optics at different wavelengths for different applications.

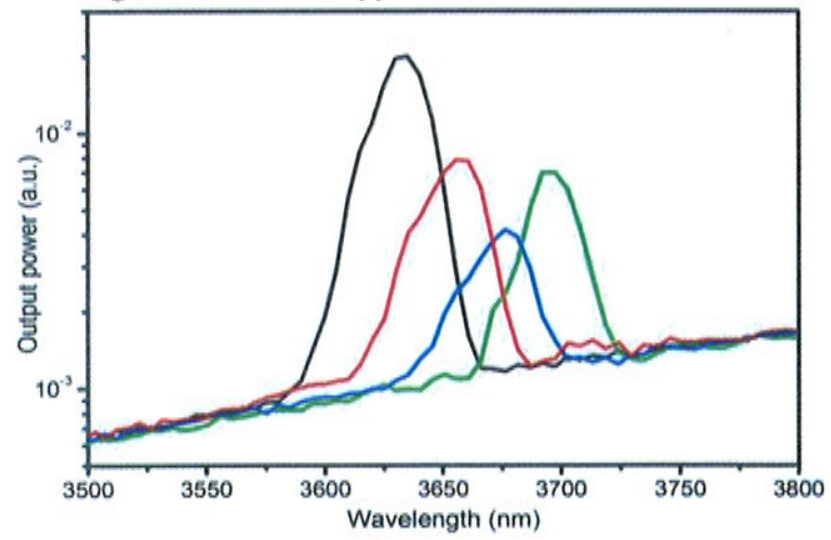

Fig. 1. Down converted signals of respectively a telecom signal at $1565 \mathrm{~nm}$ (black), $1559 \mathrm{~nm}$ (red), $1554 \mathrm{~nm}$ (blue), and $1550 \mathrm{~nm}$ (green) in a telecom wavelength translator. 


\section{I. SILICON BASED MID-IR DEVICES}

The (crystalline) silicon devices presented here are based on waveguides fabricated on a $200 \mathrm{~mm}$ silicon-on-insulator wafer with a $400 \mathrm{~nm}$ thick device layer. The relatively thick layer allows for operating the waveguides at mid-ir wavelengths. Contrary to waveguides fabricated in "standard" $220 \mathrm{~nm}$ thick layers they are not in cut-off nor leak towards the silicon substrate at these long wavelengths. Furthermore, the nonlinear parameter is found to be $-40\left(\mathrm{Wm}_{\mathrm{m}}^{-1}\right.$ at a wavelength of about $2200 \mathrm{~mm}$. A first demonstration of the efficient nonlinear interactions of these waveguides is a so called wavelength translator $[2,3]$. This translator can link the telecom band with the mid-infrared wavelength region. For example, we show that a pump centered at $2190 \mathrm{~nm}$, can down convert signals over more than an octave from the telecom band to $-3700 \mathrm{~nm}$. In a second experiment, a waveguide is used to generate an octave spanning frequency comb. In the experiment a $1600 \mathrm{~mm}$ wide waveguide is pumped with is pulses [4]. The is puises centered at $2290 \mathrm{~nm}$ are broadened in the waveguide. The output spectrum, a supercontinuum, (see Figure 2) covers an octave sparning spanning from $\sim 1550$ nim up to $-3250 \mathrm{~nm}$. Moreover, the coherence of the obtained supercontinutum is verified by beating it with a narrow linewidth CW laser on a fast photodiode. The obtained beat notes have a linewidth in the order of $50 \mathrm{kHz}$, limited by the instabilitics of the pump optical parametric oscillator. The narrow bandwidth beatnotes show that the supercontinuum consists out of a set of narrow line width lines and is as such a fiequency comb.

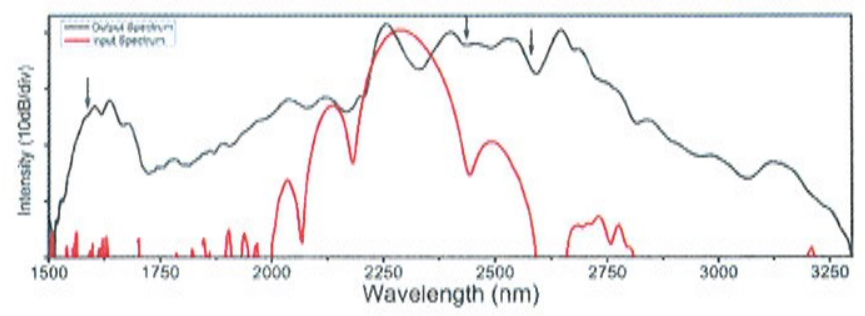

Fig. 2. The imput and output spectrum of the short pulses travelling through the $1 \mathrm{~cm}$ long silicon waveguide. The arrows indicate at which wavelength the coherence is measured

\section{NON CRYSTALLINE SILICON INTEGRATED PI.ATFORMS FOR ALL-OPTICAL SIGNAL PROCESSING}

The detrimental nonlinear absorption in silicon waveguides at telecom wavelengths hampers efficient nonlinear interactions. For all-optical signal processing applications one wants to work at telecom wavelengths. So other materials are sometimes preferred over crystalline silicon for these applications. These materials should have a wide band-gap to avoid the two photon absorption. Ideally, they should also have a high lineal and nonlinear refractive index. The combination allows for high confinement waveguides made out of a material with a high nonlinear refractive index. We have explored the use of hydrogenated amorphous silicon [5] and InGaP [6] for making highly nonlinear waveguides. Because of the relatively wide band-gap of these materials, the fabricated structures show no or only weak two photon absorption. Nonetheless, the waveguides show a strong nonlinear response. Figure 2 shows a cross section of a fabricated InGaP waveguide. As can be seen in the figure the dimensions of the waveguide $(250 \mathrm{~nm}$ high, $630 \mathrm{~nm}$ wide) are very similar than those for crystalline silicon waveguides at telecom wavelengths.

\section{STRONG PHOTON-PHONON COUPLING IN CRYSTALLINE SILICON WAVEGUIDES}

Strong photon-phonon coupling has been demonstrated in integrated structures such as photonic crystals before. Lately, however, the photon-phonon coupling has been investigated in silicon waveguides as well [7]. In a first demonstration researchers described the coupling between photons in a waveguide and phonons in a silicon nitride membrane adjacent to the waveguide. Recently, we have observed even stronger photon-phonon coupling by confining the photons and phonons in the same photonic wire [8]. By doing so we achieve a nonlinear interaction which can be stronger than the interactions arising from the nonlinear refractive index of silicon. We show a strong interaction of light and sound with frequencies around $10 \mathrm{GHz}$. Although the bandwidth of the interaction is much smaller than the Kerr effect, the scheme can be used for Radio Frequency signal processing.

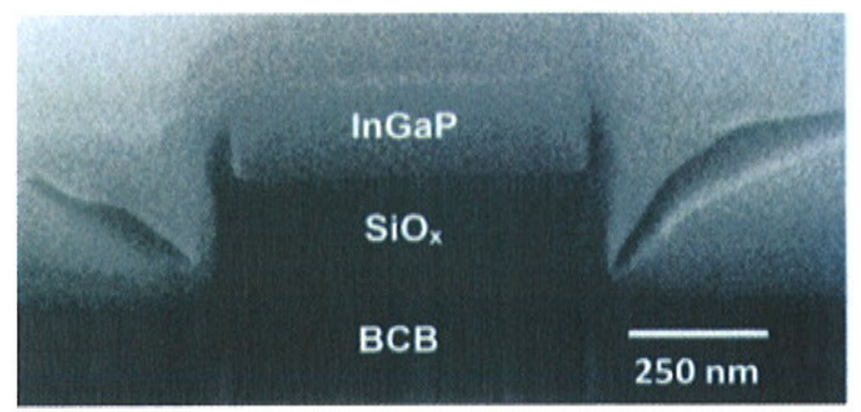

Fig. 3. A SEM cross-section of an InGaP waveguide with measured dimensions of $630 \mathrm{~nm} \times 250 \mathrm{~nm}$

\section{CONCLUSION}

In short, the high optical nonlinear response of integrated semi-conductor waveguides allows for making complex devices in the mid-infared such as an optical wavelength translator as well as an octave spanning mid-infrared frequency comb. Despite the success of (crystalline) silicon platforms, InGaP and a-Si:H platfroms are more suitable for doing ultrafast signal processing in the telecom band. Lastly, by tailoring the strong phonon-photon coupling new applications such as novel RF filters are within reach

\section{REFERENCES}

[1] J. G. Crowder, et al., "Infrared methods for gas detection," in MidInfrared Semiconductor Optoelectronics. New York: Springer-Verlag, 2006 . 
[2] X. Litu. et al. "Bridging the mid-infrared-to-telecom gap with silicon nanophotone spectral translation," Nature Photonics 6, 667-669 (2012)

[3] B Kuyken el al. "Generation of 36 um radiation and telecom-band amplification by four-wave mixing in a silicon waveguide with normal group velocity dispersion, Optics Express (2014)

[4] B. Kuyken, et al, "An octave spanning mid-infrared frequency comb generated in a silicon nanophotonic wire waveguide," Nature Communications $6,6310(2014)$

[5] B Kuyken, et al., Nonlunear properties of and nonlunear processing in hydrogenated amorphous silicon waveguides", Optics Express. 19. p. B146-B153 (20,1)

[6] (1) Dave et al "Nonimear properties of dispersion engineered InGaP photonio wire Waveguides in the telecommunication wavelength range" Optics Lexpress 23. p $4050(2015)$

[7] Shun, Hecdeuk, et al "Talorable stmmulated Brilloum scattering in namoseate silicon waveguides "Nature communcations 4 (2013)

|8| $R$ Van lacr, et al Interaction between light and highly contined hypersound in a silicon photonie nanowire", Nature Photonics 9, 199$203(2015)$ 